\title{
Time-series 3D Building Change Detection Based on Belief Functions
}

\author{
Jiaojiao Tian \\ Remote Sensing Technology Institute \\ German Aerospace Center \\ Oberpfaffenhofen, Germany 82234 \\ Email: jiaojiao.tian
}

\author{
Jean Dezert \\ ONERA - The French Aerospace Lab \\ Chemin de la Hunière \\ Palaiseau, France F-91123 \\ Email: jean.dezert@onera.fr
}

\author{
Rongjun Qin \\ The Ohio State University \\ Columbus \\ USA OH43210 \\ Email: qin.324@osu.edu
}

\begin{abstract}
One of the challenges of remote sensing image based building change detection is distinguishing building changes from other types of land cover alterations. Height information can be a great assistance for this task but its performance is limited to the quality of the height. Yet, the standard automatic methods for this task are still lacking. We propose a very high resolution stereo series data based building change detection approach that focuses on the use of time series information. In the first step, belief functions are explored to fuse the change features from the 2D and height maps to obtain an initial change detection result. In the second step, the building probability maps (BPMs) from the series data are adopted to refine the change detection results based on Dempster-Shafer theory. The final step is to fuse the series building change detection results in order to obtain a final change map. The advantages of the proposed approach are demonstrated by testing it on a set of time series data captured in North Korea.
\end{abstract}

Keywords-Change detection, belief functions, DST, DSM.

\section{INTRODUCTION}

Building change detection is one of the fundamental remote sensing research topics. Although many approaches are available, it is still very difficult to select one standard approach that works for all situations. Especially along with the improvement of the image resolution, besides building changes many irrelevant changes may also be visible in the remote sensing images, which makes the $2 \mathrm{D}$ building change detection more challenge. 3D building change detection has gained a great attention and is able to provide more accurate results. Due to the unprecedented technology development of sensor, platforms and algorithms for 3D data acquisition and generation, the $3 \mathrm{D}$ data become more accessible than before. Stereo time series data will allow a better understanding of the building change types and further increase the change accuracy.

Many research works have proved the advantages of introducing Digital Surface Models (DSM) to building change detection [1][2]. However, the performance of the 3D change detection approaches rely heavily on the quality of the DSMs. And the DSMs from satellite images do not always provide reliable height information, due to the occlusion and matching errors. In the case of large regions have incorrect height values, it is very difficult to avoid false detections. In our previous research, time-series information worked well to to improve the building detection results. In this research, we will further adopt this information to improve the change detection results.
In paper [3], the belief functions introduced in the Dempster-Shafer Theory (DS) [4] [5], and extended in DezertSmarandache Theory (DSmT) [6] were used to deal with the uncertainty information delivered from the DSMs. In [3] the possibility of using Dempter's fusion rule and the Proportional Conflict Redistribution Rule \#6 (PCR6) of DSmT in our application were tested. Though improvements have been proven by comparing with the method stated in [7], the results delivered under DS and DSmT frameworks were rather similar. Therefore, in this paper, only the DS fusion rule is used to get an initial change detection result.

This paper is organized as follow: firstly, the belief functions and building change detection fusion models are briefly reviewed. Then, the series image based fusion model together with the building extraction method are introduced. In the end, these refined fusion models are tested on the satellite real data.

\section{DS BELIEF FUNCTION BASED BUILDING CHANGE DETECTION}

\section{A. Basics of DST}

Dempster-Shafer fusion theory (DST) is one of the fundamental decision fusion theory. It allows the combination of evidence from individual experts or any data sources. The general introduction of of DST can be found in [4], [6] and[8].

Let $\Theta$ be a frame of discernment of a problem under consideration. $\Theta=\left\{\theta_{1}, \theta_{2}, \ldots, \theta_{N}\right\}$ consists of a list of $N$ exhaustive and mutually exclusive elements $\theta_{i}, i=1,2, \ldots, N$. Each $\theta_{i}$ represents a possible state related to the problem we want to solve. The assumption of exhaustivity and mutual exclusivity of elements of $\Theta$ is classically referred as Shafer's model of the frame $\Theta$. A basic belief assignment (BBA) also called a belief mass function (or just a mass for short), is a mapping $m():. 2^{\Theta} \rightarrow[0,1]$ from the power set ${ }^{1}$ of $\Theta$ denoted $2^{\Theta}$ to $[0,1]$, that verifies [4]:

$$
m(\emptyset)=0 \quad \text { and } \quad \sum_{X \in 2^{\Theta}} m(X)=1 .
$$

$m(X)$ represents the mass of belief exactly committed to $X$. An element $X \in 2^{\Theta}$ is called a focal element if and only if $m(X)>0$. In DST, the combination (fusion) of several independent sources of evidences is done with Dempster-Shafer ${ }^{2}$

\footnotetext{
${ }^{1}$ The power set is the set of all subsets of $\Theta$, including empty set.

${ }^{2}$ Although the rule has been proposed originally by Dempster, we call it Dempster-Shafer rule because it has been widely promoted by Shafer in DST.
} 
(DS) rule of combination, assuming that the sources are not in total conflict ${ }^{3}$. DS combination of two independent BBAs $m_{1}($.$) and m_{2}($.$) , denoted symbolically by D S\left(m_{1}, m_{2}\right)$, are defined by $m^{D S}(\emptyset)=0$, and for all $X \in 2^{\Theta} \backslash\{\emptyset\}$ by:

$$
m^{D S}(X)=\frac{1}{1-K^{D S}} \sum_{\substack{X_{1}, X_{2} \in 2^{\Theta} \\ X_{1} \cap X_{2}=X}} m_{1}\left(X_{1}\right) m_{2}\left(X_{2}\right),
$$

where the total degree of conflict $K^{D S}$ is given by

$$
K^{D S} \triangleq \sum_{\substack{X_{1}, X_{2} \in 2 \\ X_{1} \cap X_{2}=\emptyset}} m_{1}\left(X_{1}\right) m_{2}\left(X_{2}\right)
$$

\section{B. Building change detection}

1) Choice of the frame of discernment: As noted above, the accuracy of $2 \mathrm{D}$ change detection is limited due to the misdetections caused by irrelevant changes. These irrelevant changes have a greater effect on very high resolution (VHR) images since more detail on land-cover objects is visible. To solve this problem, in the decision fusion based 3D change detection framework, three classes have been considered. They are,

$$
\begin{gathered}
\Theta=\left\{\theta_{1} \triangleq \text { Pixel } \in \text { BuildingChange },\right. \\
\theta_{2} \triangleq \text { Pixel } \in \text { OtherChange }, \\
\left.\theta_{3} \triangleq \text { Pixel } \in \text { NoChange }\right\}
\end{gathered}
$$

and

$$
\theta_{1} \cap \theta_{2} \cap \theta_{3}=\varnothing .
$$

Based on the three classes, the set of focal elements $F E$ that are of interest in our application is:

$$
F E=\left\{\theta_{1}, \theta_{2}, \theta_{3}, \theta_{1} \cup \theta_{2}, \theta_{2} \cup \theta_{3}, \theta_{1} \cup \theta_{2} \cup \theta_{3}\right\} .
$$

Two change indicators, one from images and one from DSMs were involved in the fusion model. Changes from spectral images are highlighted by using the Iteratively Reweighted Multivariate Alteration Detection (IRMAD) [9]. Consequently height changes from DSMs are shown after robust height differencing [7].

2) BBAs construction: In [3], the sigmoidal model for both concordance and discordance indexes are constructed by projecting the change values to a sigmoid curve $f_{\tau, T} . T$ represents the symmetry point of the sigmoid curve, while the $\tau$ control the slope of it. The concordance index measures the concordance of change indicator and BBA in the assertion, while the discordance measures the opposition of change indicator to the BBAs in the assertion. The symmetry point of the concordance and discordance sigmoid curves can be automatically calculated with multi-level thresholding method proposed by Otsu [10].

Thus, using height change index as example, the BBAs for discordance and concordance height change index are functions of values $a_{\Delta H}$ and $b_{\Delta H}$ defined by

$$
a_{\Delta H}=f_{\tau, T_{1}}(\Delta H), \quad \text { and } \quad b_{\Delta H}=f_{-\tau, T_{2}}(\Delta H) .
$$

\footnotetext{
${ }^{3}$ Otherwise DS rule is mathematically undefined because of $0 / 0$ indeterminacy.
}

TABLE I: BBA construction for height change indicator $\Delta H$. $\left[K_{\Delta H}=a_{\Delta H} b_{\Delta H}\right]$

\begin{tabular}{|c|c|c|c|}
\hline Focal Elem. & $m_{1}()$. & $m_{1}^{\prime}()$. & $m_{1}^{D S}()$. \\
\hline$\theta_{1}$ & $a_{\Delta H}$ & 0 & $\frac{a_{\Delta H}\left(1-b_{\Delta H}\right)}{1-K_{\Delta H}}$ \\
\hline$\theta_{2}$ & 0 & 0 & 0 \\
\hline$\theta_{3}$ & 0 & 0 & 0 \\
\hline$\theta_{1} \cup \theta_{2}$ & 0 & 0 & 0 \\
\hline$\theta_{2} \cup \theta_{3}$ & 0 & $b_{\Delta H}$ & $\frac{(1-a \Delta H)^{b} \Delta}{1-K \Delta H}$ \\
\hline$\theta_{1} \cup \theta_{2} \cup \theta_{3}$ & $1-a_{\Delta H}$ & $1-b_{\Delta H}$ & $\frac{(1-a \Delta H)(1-b \Delta H)}{1-K \Delta H}$ \\
\hline
\end{tabular}

The factor $\tau$ could be calculated with a sample value $\left(\Delta H=1, a_{\Delta H}=0.1\right)$, which means 1 meter height change indicates $10 \%$ probability to be building changes. The BBAs for discordance and concordance image change index are built similarly. Differences appearing in 2D images give a concordance indication for all changes, which include the building changes and other changes $\left(\theta_{1} \cup \theta_{2}\right)$. In this paper the changes from images are named $\Delta I m g$.

In [3], the fusion models have been described in detail. Here we only explain the fusion model of the height changes as an example. In Table I the construction of the BBAs from the sources of evidence based DS rule of combination for the height change indicator (i.e. the first source of evidence). In Table I, $m_{1}($.$) and m_{1}^{\prime}($.$) represent the concordance and$ discordance BBAs from $\Delta H$.

\section{TIME SERIES FUSION MODEL}

To further improve the accuracy of the change detection map, the pre- and post-event building probability maps are introduced to the decision fusion model. The building probability maps are prepared using our previous research results.

\section{A. Time-series based building probability map extraction}

The building extraction method based on spatiotemporal inferences are adopted to prepare the building probability maps (BPM) [11]. The approach is mainly composed of three steps: (1) training sample selection; (2) feature extraction and classification; (3) spatiotemporal based BPM refinement.

1) training sample selection: Training sample selection is a time consuming and tedious process, which should be avoided for the automatic image processing chain. In this step, we are trying to produce the training data automatically from history database. More precisely, only one set of training data containing of building, ground\&road, shadow and trees was manually annotated. Training data for the images captures of other dates can be automatically generated by using a decision based change detection approach. The normalized DSM is used to separate the above ground object from ground and and road. And the height changes, shadow index changes and the normalized difference vegetation index (NDVI) changes are used to generate a coarse change maps, thus update the training data. More details are described in paper [11].

2) feature extraction and classification: Based on the training data, the Random Forests (RF) [12] supervised classifier was adopted. The features extracted for the classification task include: 1) Principal component analysis [13] transformation 
components of the multispectral channels; 2) Differential morphological profile [14] of the panchromatic image; 3) Normalized DSM. The RF classifier took the normalized feature vectors as input for a pixel-based classification. Besides the final classification results, the confidence values of each class label were given to each pixel. Thus a BPM map were generated for each dataset among the time-series images.

3) Spatiotemporal based BPM refinement: As it was mentioned in [11], the BPMs across all dates may be not consistent due to some potentially imprecise training samples, or unwanted objects on optical images, such as cloud/snow covered regions. In addition, as a general drawback of the pixel-based classification approaches the salt-and-pepper effect exists in the results. Thus a consistency check through spatial and temporal domain would be helpful to improve the final result. The basic idea of this approach could be explained through Eq. (8).

$$
\begin{aligned}
& P_{f}(x, y, t)= \frac{1}{\sum w(x, y, t)} \times \\
& \sum_{m=x-l}^{m=x+l} \sum_{n=y-l}^{n=y+l} \sum_{k=1}^{h} w(m, n, k) P(m, n, t)
\end{aligned}
$$

where $P(m, n, t)$ is the BPM at time $t$. The refined BPM is recorded as $P_{f}(x, y, t)$. A window size $(2 \times l+1)^{2}$ is used for the spatial consistence check. We have used $l=7$ in [11]. $h$ is the number of temporal data set. $w(m, n, k)$ is the $3 \mathrm{D}$ adaptive kernel, which aims to balance the similarity and distance of the neighboring pixels in three dimensions.

\section{B. DS based change map refinement}

One of the main advantages of DST lies in the handling indicators from various sources flexibly. Benefit from the previous steps, the refined BPMs from each time would be delivered. And a building change probability map can be calculated by using the approach from Section II. Based on the building change detection approach, we obtain a building change probability map in which all pixels represent a probability that pixel were classified as building change. Thus, when comparing two datasets the available indicators would be,

- $\quad$ pre-event BPM $\left(P_{\text {pre }}\right)$

- $\quad$ post-event BPM $\left(P_{\text {after }}\right)$

- $\quad$ initial building change probability map $\left(P_{B C}\right)$

To model this situation more precisely, we categorize the change situations into four groups, which are buildings to buildings ( $\mathrm{BB})$, non-building to buildings (NB), buildings to non-building (BN) and non-building to non-building (NN). Based on these four classes and the indicators, the $F E$ set that are of interest in this fusion model is,

$$
F E=\{B B, N B, B N, N N, B B \cup B N, B B \cup N B, \Theta\}
$$

The probability masses $\left\{P_{1}, P_{2}, P_{3}\right\}$ obtained respectively from these three indicators are assigned to $F E$ as shown in Table. II. One of the basis principles is that all newly built buildings should have a lower value in the pre-event BPM, and a higher value in the post-event BPM. Based on the DS fusion rule, the fused masses are listed in the last column.

TABLE II: DS fusion model for result refinement $\quad[K=$ $\left.P_{1} * P_{3}\right]$.

\begin{tabular}{|c|c|c|c|c|}
\hline FE. & $P_{\text {pre }}$ & $P_{\text {after }}$ & $P_{B C}$ & Fused mass \\
\hline$B B$ & 0 & 0 & 0 & $\frac{P_{1} * P_{2} *\left(1-P_{3}\right)}{1-K}$ \\
\hline$N B$ & 0 & 0 & $P_{3}$ & $\frac{\left(1-P_{1}\right) * P_{3}}{1-K}$ \\
\hline$B N$ & 0 & 0 & 0 & 0 \\
\hline$N N$ & 0 & 0 & 0 & 0 \\
\hline$B B \cup B N$ & $P_{1}$ & 0 & 0 & $\frac{P_{1} *\left(1-P_{2}\right) *\left(1-P_{3}\right)}{1-K}$ \\
\hline$B B \cup N B$ & 0 & $P_{2}$ & 0 & $\frac{\left(1-P_{1}\right) * P_{2} *\left(1-P_{3}\right)}{1-K}$ \\
\hline$\Theta$ & $1-P_{1}$ & $1-P_{2}$ & $1-P_{3}$ & $\frac{\left(1-P_{1}\right) *\left(1-P_{2}\right) *\left(1-P_{3}\right)}{1-K}$ \\
\hline
\end{tabular}

\section{Time-series fusion model}

The previous fusion steps are performed to each multitemporal data pair separately. They can be then combined in the time-series fusion model. In this section, we use three datasets captured from three dates as an example to describe our fusion model. Three datasets are notated as $d_{1}, d_{2}$ and $d_{3}$, respectively. They are arranged according to the acquisition time. $d_{1}$ is the oldest dataset. Then the building change detection (BC) outcomes among these datasets can be recoded as $B C_{12}, B C_{23}$ and $B C_{13}$, respectively.

By referring to the fusion model in Table II, a global $\operatorname{mass}\left\{m_{B B}, m_{N B}, m_{B N}, m_{N N}, m_{B B \cup B N}, m_{B B \cup N B}, m_{\Theta)}\right\}$ can be obtained. We will transform the global mass to a threeclasses $F E, F E=\{N B, \overline{N B}$, and $\Theta\}$ by coarsening the original set of focal elements. For this, we apply the following transformation which can be seen as a partial pignistic transformation. In the pignistic probabilities [4], global masses of joint elements are averagely redistributed to each class. Since the full ignorance $\Theta$ is one focal elements in the three-classes $F E$, we will keep this value to $m(\Theta)$ and only take the partial ignorance when calculating the $m^{\prime}($.$) as shown in Eq. (10).$

$$
\begin{aligned}
& m^{\prime}(N B)=m_{N B}+\frac{1}{2} m_{B B \cup N B} \\
& m^{\prime}(B B)=m_{B B}+\frac{1}{2} m_{B B \cup N B}+\frac{1}{2} m_{B B \cup B N}, \\
& m^{\prime}(B N)=m_{B N}+\frac{1}{2} m_{B B \cup B N} \\
& m^{\prime}(N N)=0 \\
& m^{\prime}(\Theta)=m(\Theta)
\end{aligned}
$$

To clarify the notation, we use $a, b$ and $c$ to represent the mass values for $N B, \overline{N B}$ and $\Theta$, respectively. With $m^{\prime}(N N)=0$, they can be calculated with Eq. (11). Thus the building change detection results from $N B_{12}, N B_{23}$ and $N B_{13}$, denoted as $P_{N B 12}, P_{N B 23}$ and $P_{N B 13}$, can be fused according to the fusion model shown in Table. III. In this fusion model, the focal three change classes would be, changes happened between $d_{1}$ and $d_{2}$, notated as $C_{1}$; changes happened between between $d_{2}$ and $d_{3}$, notated as $C_{2}$; and no-building change, notated as $C_{3}$. 


$$
\begin{aligned}
& a=m^{\prime}(N B), \\
& c=m^{\prime}(\Theta), \\
& b=m^{\prime}(\overline{N B})=1-m^{\prime}(N B)-m^{\prime}(\Theta) .
\end{aligned}
$$

TABLE III: The indicators for time series fusion model

\begin{tabular}{|c|c|c|c|}
\hline FE. & $P_{N B 12}$ & $P_{N B 23}$ & $P_{N B 13}$ \\
\hline$C_{1}$ & $P_{1 a}$ & 0 & 0 \\
\hline$C_{2}$ & 0 & $P_{2 a}$ & 0 \\
\hline$C_{3}$ & 0 & 0 & $P_{3 b}$ \\
\hline$C_{1} \cup C_{2}$ & 0 & 0 & $P_{3 a}$ \\
\hline$C_{2} \cup C_{3}$ & $P_{1 b}$ & 0 & 0 \\
\hline$C_{1} \cup C_{3}$ & 0 & $P_{2 b}$ & 0 \\
\hline$\Theta$ & $P_{1 c}$ & $P_{2 c}$ & $P_{3} c$ \\
\hline
\end{tabular}

With the conjunction rules, the following belief masses will be obtained,

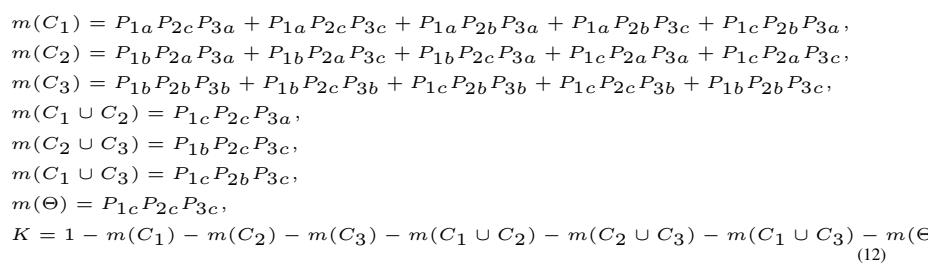

in which $K$ represents the mass of conflict. Based on the DS fusion rule, the final mass will be calculated by

$$
m_{D S}(X)=\frac{m(X)}{1-K}
$$

TABLE IV: Time series datasets description

\begin{tabular}{|c|c|c|c|c|}
\hline No. & Satellite & Capture date & \multicolumn{2}{|c|}{ Resolution $(\mathrm{m})$} \\
\cline { 4 - 5 } & & & PAN & MS \\
\hline 1 & IKONOS & $23-02-2006$ & 1 & 4 \\
\hline 2 & GeoEye-1 & $20-12-2009$ & 0.5 & 2 \\
\hline 3 & IKONOS & $12-01-2010$ & 1 & 4 \\
\hline 4 & IKONOS & $13-05-2010$ & 1 & 4 \\
\hline 5 & IKONOS & $07-01-2011$ & 1 & 4 \\
\hline 6 & IKONOS & $02-05-2011$ & 1 & 4 \\
\hline
\end{tabular}

\section{B. Results and evaluation}

In the first change detection step, the data from 2006 are used as the pre-event test data. The rest five datasets are the post-event dataset. Thus five change detection case studies are prepared and named as $C_{06-09}, C_{06-1001}, C_{06-1005}$, $C_{06-1101}, C_{06-1105}$, correspondingly. The change indicators from DSMs and images are detected respectively by using robust height differences and IRMAD. The change maps are recorded as $H_{\text {diff }}$ and $I m g_{\text {diff }}$. At the same time, BPMs from all six datasets are calculated and refined with the approach described in section 3.1. Then the change detection result between each pairs of datasets is refined by using the preevent and post-event BPMs.

To evaluate quantitatively the performances of the different fusion approaches, the extracted BBAs from both approaches (original and refined) are compared to the manually extracted change reference masks. The results are analyzed in terms of Receiver Operating Characteristic (ROC) curve [16]. A larger area under the ROC curve (AUC) indicates a better accuracy of the building change map. The numerical evaluation results are described in Table V. The obtained AUC values prove an obvious accuracy improvement after the proposed fusion model is applied. The $m^{\prime}(N B)$ is used as the first-step change detection results, and listed as Re fined $_{1}$.

, for $X \in\left\{m_{B B}, m_{N B}, m_{B N}, m_{N N}, m_{B B \cup B N}, m_{B B \cup N B}, m_{\Theta)}\right\}$, TABLE V: Building change detection accuracy comparison. and $X \neq \emptyset$; and $m_{D} S(\emptyset)=0$.

\section{EXPERIMENTS}

The improved building change detection fusion models have been tested on satellite images. The datasets and the experiments are described in this section.

\section{A. Datasets}

The experimental datasets consist of five pairs of IKONOS and one pair of GeoEye-1 stereo imagery captured from 2006 to 2011. The detailed capture dates of these data are shown in Table IV. The true color images of the earliest and latest datasets are shown in Fig. 1 (a) and 1 (b), respectively. Within these five years, many new buildings are constructed in this test region. As a data preparation procedure, DSMs have been generated based on the method explained in [15].

The sub-pixel co-registration among these data is performed based on the camera model parameters correction [11]. The radiometric co-registration method is described in [7].

\begin{tabular}{|c|c|c|c|c|c|}
\hline Change maps & $C_{06-09}$ & $C_{06-1001}$ & $C_{06-1005}$ & $C_{06-1101}$ & $C_{06-1105}$ \\
\hline$H_{\text {diff }}$ & 0.9267 & 0.9233 & 0.9016 & 0.8289 & 0.8211 \\
\hline Img $_{\text {diff } f}$ & 0.9049 & 0.5937 & 0.9004 & 0.8283 & 0.8610 \\
\hline Fusion & 0.9540 & 0.9271 & 0.9474 & 0.8885 & 0.8862 \\
\hline Refined $_{1}$ & 0.9771 & 0.9744 & 0.9668 & 0.9241 & 0.9442 \\
\hline
\end{tabular}

In the time-series fusion model, we have tested the data from 2006, 2009 and 2010 May as a test combination. The further improved building change probability map $\left(C_{06-09}\right)$ with $(\mathrm{AUC}=0.9795)$ is delivered. The differences between the original change detection result and the refined one can be observed in Fig. 2. 


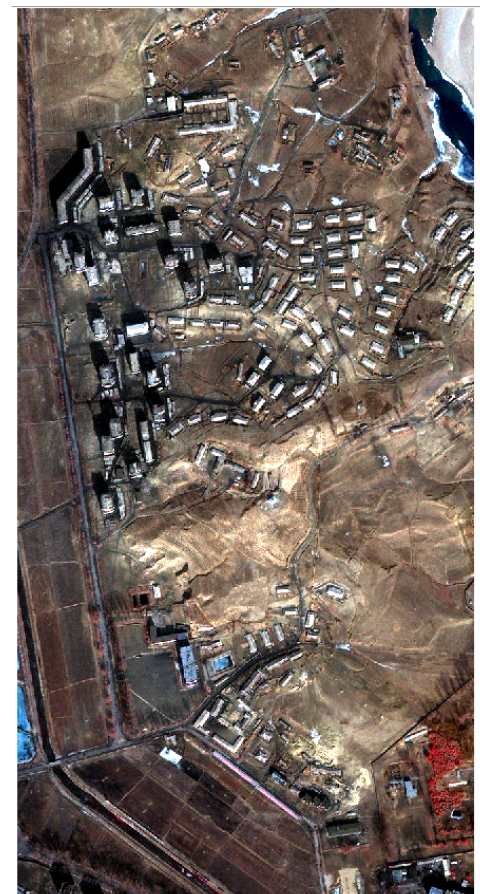

(a)

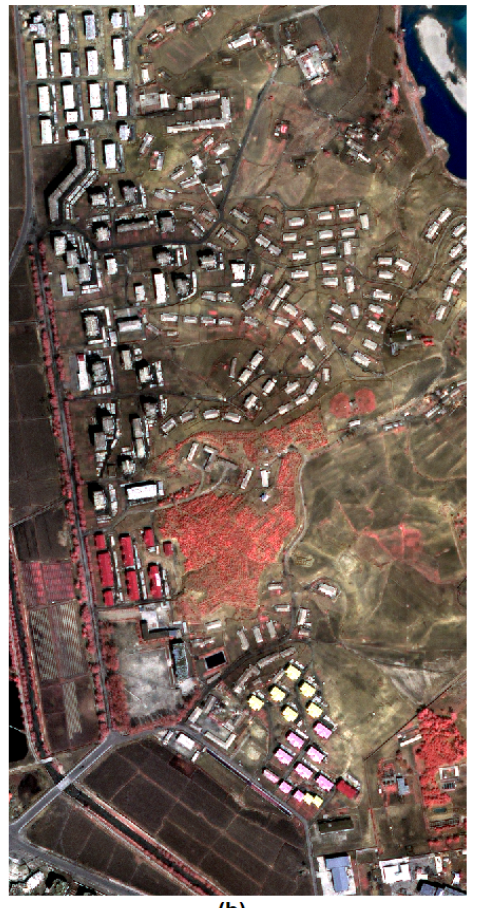

(b)

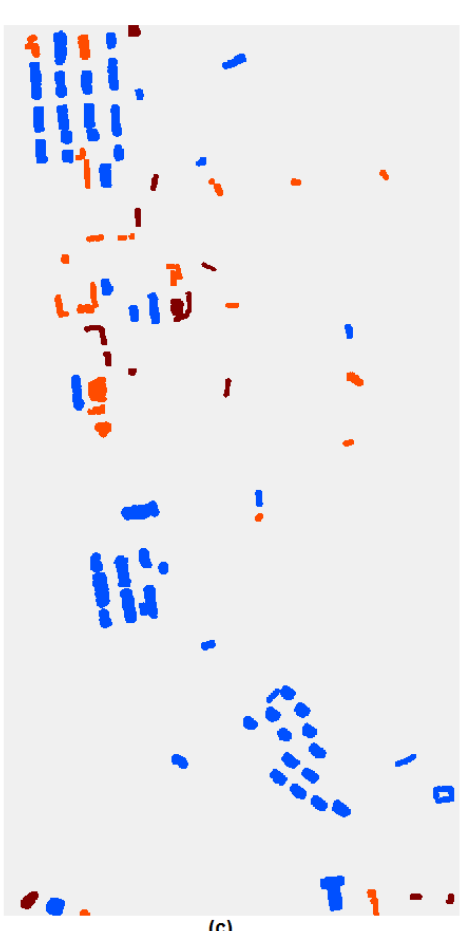

(c)

Fig. 1: The true color images of the first (a) and sixth (b)experimental dataset and (c) the change reference map (Blue: built before 2009; Orange: built before January 2011; Red: built before May 2011)

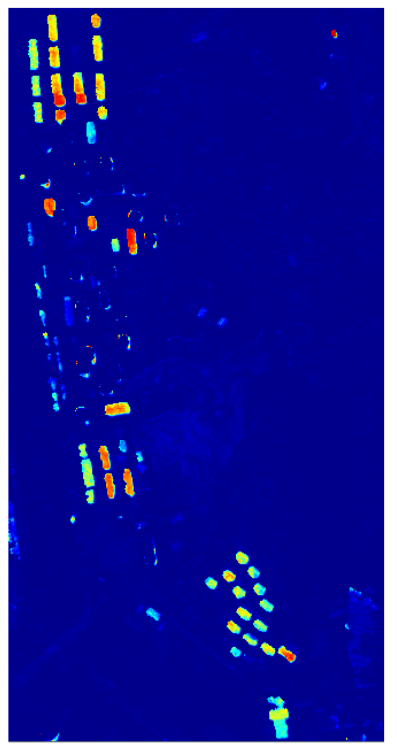

(a)

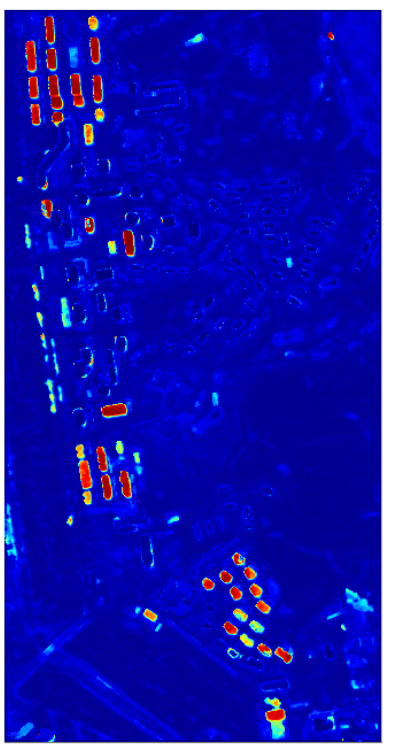

(b)

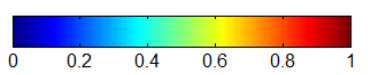

Fig. 2: The building change detection results between 2006 and 2009 based on (a) inital fusion model (b) time-series fusion model.

\section{CONCLUSIONS}

Detecting building changes is an important but difficult topic. Many approaches have been proposed for specific build- ing types or for certain types of data sets. In addition, the image quality and the existing of some unwanted objects may also influence the effectiveness of some approaches. Our previous research has evidenced the performance of the belief functions in DSM assisted change detection [3]. In this paper, we have further explored in more detail the belief functions for building change detection. Time-series data were used for this purpose. They were firstly adopted to provide the BPMs after checking the temporal consistency for each date. Then, pre- and postevent BPMs are used to improve the accuracy of the DS-based building detection result. In the last step, the time-series change detection results can be fused again according to the DS fusion rule, which results a further improved building change map. However, in the time-series fusion model, only three sets of data are involved. As part of our further work, this fusion model will be further refined that may accommodate more datasets as inputs.

\section{REFERENCES}

[1] R. Qin, J. Tian, and P. Reinartz, "3d change detection-approaches and applications," ISPRS Journal of Photogrammetry and Remote Sensing, vol. 122, pp. 41-56, 2016.

[2] J. Tian, P. Reinartz, P. d'Angelo, and M. Ehlers, "Region-based automatic building and forest change detection on cartosat-1 stereo imagery," ISPRS J. Photogramm. and Remote Sens., vol. 79, pp. 226239, 2013.

[3] J. Tian, P. Reinartz, and J. Dezert, "Building change detection in satellite stereo imagery based on belief functions," in Urban Remote Sensing Event (JURSE), 2015 Joint. IEEE, 2015, pp. 1-4.

[4] G. Shafer, A mathematical theory of evidence. Princeton university press Princeton, 1976. 
[5] A. Dempster, "Upper and lower probabilities induced by a multivalued mapping," The Annals of Mathematical Statistics, vol. 38, no. 2, pp. 325-339, 1967.

[6] F. Smarandache and J. Dezert, Advances and Applications of DSmT for Information Fusion. American Research Press, Rehoboth, NM, U.S.A, 2004-2015, vol. 1-4. [Online]. Available: http://www.onera.fr/staff/jean-dezert?page $=2$

[7] J. Tian, S. Cui, and P. Reinartz, "Building change detection based on satellite stereo imagery and digital surface models," IEEE Trans. Geosci. Remote Sens., vol. 52, no. 1, pp. 406-417, 2014.

[8] J. Dezert and A. Tchamova, "On the validity of Dempster's fusion rule and its interpretation as a generalization of Bayesian fusion rule," Int. J. Intell. Syst., vol. 29, no. 3, pp. 223-252, 2014.

[9] A. A. Nielsen, "The regularized iteratively reweighted MAD method for change detection in multi-and hyperspectral data," IEEE Trans. Image Process., vol. 16, no. 2, pp. 463-478, 2007.

[10] N. Otsu, "A threshold selection method from gray-level histograms," IEEE Trans. Syst., Man, Cybern., vol. 9, no. 1, pp. 62-66, 1975.

[11] R. Qin, J. Tian, and P. Reinartz, "Spatiotemporal inferences for use in building detection using series of very-high-resolution space-borne stereo images," International Journal of Remote Sensing, vol. 37, no. 15, pp. 3455-3476, 2016.

[12] L. Breiman, "Random forests," Machine learning, vol. 45, no. 1, pp. 5-32, 2001.

[13] I. T. Jolliffe, "Principal component analysis and factor analysis," in Principal component analysis. Springer, 1986, pp. 115-128.

[14] J. A. Benediktsson, M. Pesaresi, and K. Amason, "Classification and feature extraction for remote sensing images from urban areas based on morphological transformations," IEEE Transactions on Geoscience and Remote Sensing, vol. 41, no. 9, pp. 1940-1949, 2003.

[15] P. d'Angelo and P. Reinartz, "DSM based orientation of large stereo satellite image blocks," Int. Arch. Photogramm. Remote Sens. Spatial Inf. Sci, vol. 39, no. B1, pp. 209-214, 2012.

[16] M. H. Zweig and G. Campbell, "Receiver-operating characteristic (roc) plots: a fundamental evaluation tool in clinical medicine." Clinical chemistry, vol. 39, no. 4, pp. 561-577, 1993. 